\title{
Research on the Path of China Cities Service Consumption Upgrading
}

\author{
Chen Fan, Wang Shengjin* \\ Shanghai Urban Construction Vocational College, Shanghai, 201415, China
}

\begin{abstract}
Based on the panel data of "first-line cities" and "new first-line" cities from 2006 to 2018, this paper constructs an extended linear expenditure system model (ELES) to empirically study the path law of service sub-consumption upgrade. Based on the full-sample OLS estimation, it is found that the overall marginal propensity for service consumption in the sample cities is 0.418 , of which the sub-consumption propensity for cultural, entertainment and education services is the most obvious, followed by medical and health services, and transportation and communication services are the lowest. Based on service subconsumption, the research on price and income elasticity found that the expenditure and income elasticity of cultural entertainment and education, which has the most significant marginal propensity to consume, is the largest, while the price and income elasticity of health care services is the smallest. An empirical test based on sub-samples found that the marginal service consumption propensity of "first-line cities" and "new firstline cities" are 0.558 and 0.379 , respectively; in terms of service breakdown, "first-line cities" have propensity to consume cultural entertainment and education services Significantly higher than the "new first-line cities", the sub-item propensity to consume in medical and health services is slightly higher, and the sub-item propensity to consume in transportation and communication services is slightly lower.
\end{abstract}

\section{Introduction}

The consumption structure is an important manifestation of the level of economic and social development of a country or region. Judging from the consumption upgrading laws of developed countries such as the United States and Japan, the upgrading of the consumption structure has followed the trajectory of "food, housing, transportation and recreation", and service consumption has become the highest and most important link in the social consumption structure. According to Bell's "PostIndustrial Society" on socio-economic transformation and consumption upgrade according to the discussion, consumption preference has been upgraded from the general living expenditure of ordinary durable goods to the service consumption expenditure of leisure and entertainment products with the increase of national income; Chan et al. proposed a positive matching relationship between consumption upgrade and per capita national income.

According to a survey by the National Bureau of Statistics, China's per capita GDP reached US $\$ 8,826$ in 2017, which has completely exceeded the basic economic structural conditions necessary for consumption upgrades in the world. At the beginning of 2019, the National Bureau of Statistics estimated the growth trend of the middle-income group in China, predicting that the total number of middle-income groups in 2020 will exceed 300 million. This service-oriented economic and social structure has become the economic foundation for the upgrading of service consumption structure. The upgrading of consumption structure will also be an inevitable choice for China's economic and social transformation. However, in 2018, the proportion of China's urban and rural residents' service consumption has not yet reached $40 \%$, and China's overall service consumption proportion is still low.

The consumption trend and upgrade path of China's first-line cities are the frontlines of the upgrade of the consumption structure of the whole society in China. Therefore, scientific research on the law of service consumption upgrade paths in first-line cities and reveals the trend of changes in consumption structure is important for improving the consumption mechanism and enhancing consumption in China's social and economic transformation. The sustainable promotion of China's economic development is of great practical significance.

\section{Research consumption}

According to the current National Bureau of Statistics on social retail sales, the consumption of catering services included in social retail sales accounts for only a small percentage of the total service consumption, while urban and rural residents' consumption includes two major parts, commodity consumption and service consumption, respectively. As social retail the amount of statistics is the amount of physical goods and catering services in market transactions. Therefore, the social retail sales indicators

e-mail: CJ0840@succ.edu.cn 
commonly used in the study of residential consumption are no longer applicable to the study of service consumption. Based on the empirical research needs of this article and the current statistical status of the National Bureau of Statistics, it is very necessary to clarify the research calibre of consumer services and conduct statistical tracking.

This article draws on the research methods and indicator selection basis of many scholars such as Zhang Lei (2017), Yang Tianyu and others (2018) and Liu Tao and others (2019), and extends the research scope of service consumption from social retail sales to household consumption statistics. According to service consumption the connotation of non-commodity service expenditure of Chinese residents in cultural life and other aspects, and refer to the statistical calibre of the National Bureau of Statistics on residents' consumption, the specific content of the service consumption in this article is divided into eight categories: family services, transportation and communication services, clothing processing services, catering services, residential services, education, culture and entertainment services and other services. At present, the National Bureau of Statistics has not yet formed economic data on service consumption, and the eight categories of service consumption proposed in this article include residential services, household services and clothing processing services, etc. There are no official statistics, and catering services are a social retailer. The calibre and commodity consumption are combined, so it cannot truly reflect the catering service consumption. To sum up, this article defines the statistical calibre of service consumption as the three major parts of residents' consumption: transportation and communication expenditure, medical and health care expenditure, cultural entertainment and education expenditure. According to data from the National Bureau of Statistics in 2018, the main service consumption of the above three major components has accounted for $32 \%$ of household consumption, and the contribution of service consumption to GDP is as high as $61.6 \%$. The service consumption industry has become a pillar of national economic development.

Table1. Parameter estimation and statistical test results

\begin{tabular}{|c|c|c|c|c|}
\hline Samples & Coefficient & TCSC & HCSC & CCEES \\
\hline \multirow{5}{*}{ First-line citi } & $K_{i}$ & 189.00 & 187.43 & 111.12 \\
\hline & $b_{i}$ & 0.02 & 0.15 & 0.37 \\
\hline & & & & \\
\hline & $P_{i} r_{i}$ & 80.22 & 389.12 & 345.34 \\
\hline & $V_{i}$ & 168.23 & 823.32 & 236.54 \\
\hline \multirow{5}{*}{ New first-line $c$} & $K_{i}$ & 219.23 & 167.12 & 56.23 \\
\hline & $b_{i}$ & 0.02 & 0.15 & 0.29 \\
\hline & & & & \\
\hline & $P_{i} r_{i}$ & 61.23 & 310.56 & 234.56 \\
\hline & $V_{i}$ & 983.12 & 865.23 & 998.76 \\
\hline
\end{tabular}

Parameter estimation and statistical test results of distribution samples in first-tier cities are as table 1, which TCST means transportation and communication service consumption; HCSC means health care service consumption; CCEES means consumption of cultural entertainment and educational services.

\section{3 empirical analysis and results}

\subsection{ELES model estimation of mixed panel}

Using the hybrid panel OLS estimation method to fit the ELES model of service consumption in first-line cities, and substituting the results into the utility function, the sub-item basic expenditure of service consumption can be calculated. According to the analysis of the ELES model of service consumption in first-line cities, the overall marginal propensity to consume services in first-line cities 
is 0.418 , indicating that for every $1 \%$ increase in per capita disposable income in first-line cities in the sample, $41.8 \%$ will be used for service consumption expenditure. Among them, the propensity to consume cultural entertainment and education services is the most obvious.

For every $1 \%$ increase in per capita disposable income, $24.1 \%$ will be spent on cultural entertainment and education. Expenditures for this sub-consumer service account for $57.65 \%$ of all service consumption expenditures; followed by the coefficient of marginal propensity for consumption of healthcare services is 0.148 ; the marginal propensity for consumption of transportation and communication services is the lowest, only 0.029. The test results show that the consumption of residents in firstline cities is the tendency to focus on fees is very obvious, especially when the disposable income is increased for cultural entertainment, education services and medical and health care services. First of all, although compulsory education reduces the cost of education expenditure in the expenditure of education service consumption, most families in the fierce social competition have expenditures on children's off-campus training and education.

According to a Wind survey, my country's the education and training industry has maintained an average annual growth rate of over $10 \%$, and the price index of education services is also much higher than the CPI index in the same period. The expenditure of cultural and entertainment consumption is very obvious in film consumption and tourism consumption. The box office revenue of my country's film consumption has grown rapidly from 17.07 billion Yuan in 2012 to 55.911 billion Yuan in 2017, with an average annual compound growth rate of $26.78 \%$; in tourism consumption the most representative "May Day" and Spring Festival outbound visits during 2012-2017 coincided with a growth rate of $12.1 \%$. In terms of consumption of health care services, the average annual growth rate of my country's medical service expenditure and health service expenditure from 2012 to 2017 was $16.7 \%$ and $13.1 \%$, respectively.

\subsection{Sub-sample parameter estimation and statistical testing}

In the previous article, my country's first-line cities were divided into "first-line" and "new first-line" according to the scale of development. Here, two samples of "first-line" and "new first-line" are analysed using the ELES model of service consumption. The specific results are shown in Table 2. According to the parameter estimation results of sub-samples, "first-line cities" and "new first-line cities", the marginal propensity for service consumption of Beijing is 0.558 and 0.379 respectively, reflecting that the service consumption level of the four "first-line cities" in Beijing, Shanghai, Guangzhou and Shenzhen is much higher than that of the "new first-line cities". This article believes that the income level of residents in the "first-line cities" of Beijing, Shanghai, Guangzhou and Shenzhen is much higher than that of "new first-line cities" such as Tianjin and Chongqing. The consumer market "An Overview of Chinese Core Journals" is a core journal of trade economy.
The consumption expenditures of transportation and communication, culture and entertainment and other services in the country are similar. Only education and medical care are different. Therefore, there is a huge difference in the marginal propensity of service consumption expenditure between "first-line cities" and "new first-line cities". From the perspective of service consumption tendency, the consumption of cultural, entertainment and education services in "first-line cities" is significantly higher than that in "new first-line cities". The reason is that the service consumption is enjoyable consumption, and the consumption field in "first-line cities" is broader, and it has a better economic foundation, so the difference between "first-line cities" and "new firstline cities" is obvious. In terms of the propensity to consume healthcare services, the consumption propensity of "first-line cities" is slightly higher than that of "new first-line cities".

On the one hand, it shows that regardless of "first-line" or "new first-line", the expenditure on healthcare services has been paid by residents in all regions. On the other hand, it also reflects that the problem of "expensive medical care" in large cities in my country has been significantly improved as the country deepens the supply-side structural reform of the medical system.

In terms of transportation and communication service consumption, the marginal propensity to consume in "first-line cities" is slightly lower than that of "new firstline cities", reflecting that "first-line cities" have continuously improved their urban development level in addition to their huge economic scale. Consumption of transportation and communication services has a lower proportion of expenditures under the conditions of higher income levels and more developed cities. Health services (0.148), transportation and communication services are the lowest (0.029); research based on the price and income elasticity of service consumption has found that cultural, entertainment and education expenditures and income elasticity's with the most significant marginal propensity for consumption have the greatest income elasticity, and the price and income elasticity of consumption of health care services

An empirical test based on sub-samples found that the marginal propensity to consume services in "first-line cities" and "new first-line cities" were 0.558 and 0.379 , respectively. Among them, "first-line cities" had significantly higher propensity to consume cultural entertainment and education services than "new first-line cities" "The propensity to consume healthcare services in "first-line cities" is slightly higher than that of "new firstline cities" and the propensity to consume transportation and communication services in "first-line cities" is slightly lower than that of "new first-line cities. 


\section{References}

1. Jock Collins. (2003) Cultural Diversity and Entrepreneurship: Policy Responses to Immigrant Entrepreneurs in Australia.J. Entrepreneurship and Regional Development., 15:166-198.

2. Sanchez T W. (2018) The connection between public transit and employment: The cases of Portland and Atlanta. J. Journal of the American planning association., 13:66-101.

3. Kasia MotoCorp, Jani Petri Litmanen. (2010) Welfare State and Life Satisfaction: Evidence from Public Health Care.J. Economics.

4. Zonal Hisami. (2010) The Size and Composition of Government Spending in Europe and Its Impact on Well-Being.J. Kilos. 